\title{
Una comparación de los modernos sistemas de calcinación de cal
}

F. SCHWARZKOPF

Rock Products, julio de 1970, págs. 68-71, 112

La producción de cal en Estados Unidos durante el año de 1969 fue de unos 19,7 millones de toneladas y en 1970 se espera sobrepasar esta cifra. La cal tiene muchas aplicaciones en la construcción, industrias químicas y del acero, estabilización de suelos, etc. Las exigencias del producto, tanto físicas como químicas, cubren un amplio margen, y no es sorprendente que estas exigencias de calidad tan variables hayan creado gran número de sistemas diferentes de producción.

Todas las materias primas para la fabricación de la cal -aunque no muy distintas químicamente- son, esencialmente, carbonato cálcico o carbonato cálcico-magnésico (dolomita), con diferentes tipos y cantidades de impurezas. Pueden cubrir un amplio intervalo en propiedades físicas, tales como distribución granulométrica, dureza, porosidad, tamaño de cristal, contenido de humedad superficial, y no todos los sistemas de calcinación se comportan del mismo modo para cumplir las exigencias acostumbradas.

Los costos de producción que resultan del consumo de combustible, necesidades de energía, mano de obra, costos de financiación y amortización y, naturalmente, costos de primera instalación, crean nuevas limitaciones a la hora de elegir los sistemas de calcinación de la cal. Las disponibilidades actuales o futuras de combustibles y otras exigencias auxiliares para cumplir los códigos de polución atmosférica, cada vez más estrictos, son, naturalmente, nuevos factores de importancia a tener en cuenta.

A la vista de tantas variables y exigencias, en este trabajo se va a tratar de hacer un intento de clasificación de los modernos sistemas de calcinación de cal disponibles, aplicables en la industria de la cal norteamericana. El estudio comparativo se limita a seis sistemas y diversos hornos, tales como el hogar vibratorio, el hogar múltiple y otros. No se incluyen las unidades que trabajan en la industria de cal o en las plantas que fabrican cal "captive", en parte, porque se consideran de menos importancia y, en parte, porque no se dispone de datos inmediatos. La omisión de tales sistemas no es una censura de los respectivos procesos tecnológicos, costos de capital y producción o calidad de los productos obtenidos.

El desarrollo histórico de los actuales hornos de cal, a partir del descubrimiento accidental de la cal viva producido en un juego al aire libre a través de fosos, hornos de crisol y hornos de anillo hasta las unidades de gran producción sofisticados y automatizados, es de poca significación en esta comparación; sólo se considera la tecnología de hoy día. 


\section{HORNOS ROTATORIOS LARGOS}

Estas unidades son suministradas en Estados Unidos por Allis-Chalmers, Fuller-Traylor, Kennedy Van Saun, F. L. Smidth y Vulcan Iron Works. Los hornos rotatorios se encuentran en la mayor parte de las instalaciones y son, probablemente, los más versátiles. Los hay en el mercado desde pequeñas unidades, de 50 t/día, a unidades gigantes, de 1.000 t/día (estas últimas sin experiencia). Puesto que la industria del cemento trabaja hoy día satisfactoriamente con hornos de más de $6 \mathrm{~m}$ de diámetro y por encima de $180 \mathrm{~m}$ de

T A в L A 1

\begin{tabular}{|c|c|c|c|c|c|c|}
\hline Sistema & 1 & 2 & 3 & 4 & 5 & 6 \\
\hline $\begin{array}{l}\text { Intervalo de capacidad } \\
\text { t/día }\end{array}$ & $100-1.000$ & $150-1.000$ & $100-680$ & $100-600$ & $50-300$ & $200-400$ \\
\hline $\begin{array}{l}\text { Mayores unidades en } \\
\text { servicio en U.S.A. }\end{array}$ & 650 & 500 & 680 & 300 & 230 & $300(*)$ \\
\hline $\begin{array}{l}\text { Intervalo de tamaño de } \\
\text { alimentación (mm) }\end{array}$ & $6,5-51,0$ & $9,5-44,0$ & $25,4-180,0$ & $3,2-102,0$ & $0,25-6,4$ & $19,0-152$ \\
\hline $\begin{array}{l}\text { Relacción de tamaño } \\
\text { de alimentación per- } \\
\text { misible }\end{array}$ & $1: 3$ & $1: 4$ & $1: 2$ & $1: 2$ & - & $1: 3$ \\
\hline Tipo de combustible & $\begin{array}{l}\text { gas/fuel- } \\
\text { oil/carbón }\end{array}$ & $\begin{array}{l}\text { gas/fuel- } \\
\text { oil/carbón }\end{array}$ & Gas & $\begin{array}{l}\text { Gas/ } \\
\text { fuel-oil }\end{array}$ & $\begin{array}{l}\text { Gas/ } \\
\text { fuel-oil }\end{array}$ & $\begin{array}{l}\text { Gas/ } \\
\text { fuel-oil }\end{array}$ \\
\hline $\begin{array}{c}\text { Consumo de combusti- } \\
\text { ble kcal } \times 10^{3} / \mathrm{kg}\end{array}$ & $1,75-2,0$ & $1,25-1,5$ & 1,25 & $1,18-1,25$ & 1,06 & $0,88-1,0$ \\
\hline $\mathrm{CO}_{2}$ en la cal \% (**) & $0,2-0,8$ & $0,2-0,8$ & $1,0-2,5$ & $1,0-1,5$ & 0,5 & $1,0-1,5$ \\
\hline $\begin{array}{l}\text { Actividad (ASTM C 110) } \\
\text { (a) tiempo total, minu- } \\
\text { tos } \\
\text { (b) }{ }^{\circ} \text { C/minuto }{ }^{(* * *)}\end{array}$ & $\begin{array}{c}2-3 \\
5-25\end{array}$ & $\begin{array}{r}2-3 \\
5-25\end{array}$ & $\begin{array}{l}10 \\
5-8\end{array}$ & $\begin{array}{c}3 \\
10-20\end{array}$ & $\begin{array}{l}1-2 \\
20-35\end{array}$ & $\begin{array}{r}3-5 \\
6-14\end{array}$ \\
\hline $\begin{array}{l}\text { Costo del equipo por } \\
\left.\text { t/día (pesetas } \times 10^{3}\right)\end{array}$ & $105-125$ & $100-120$ & $90-110$ & $90-175$ & $140-210$ & $125-140$ \\
\hline $\begin{array}{l}\text { Cost o instalado por } \\
\text { t/día (pesetas } \times 10^{3} \text { ) }\end{array}$ & $260-280$ & $240-270$ & $175-210$ & $190-245$ & $280-420$ & 200-225 \\
\hline $\begin{array}{l}\text { Consumo de energía } \\
\mathrm{kWh} / \mathrm{t}\end{array}$ & 24-32 & $32-36$ & $10-25$ & $28-32$ & 25 & $22-25$ \\
\hline
\end{tabular}

CLAVE

1. Horno rotatorio largo.

2. Horno rotatorio corto con precalentador vertical (Kennedy Van Saun).

3. Horno vertical (Unión Carbide).

4. Hogar rotatorio (Calcimatic)

5. Lecho fluido (Dorr-Oliver).

6. Regenerador de flujo paralelo (Kennedy Van Saun).

(*) En Bélgica. No hay ninguna instalación en U.S.A.

(**) El intervalo dado es compatible con una marcha normalmente económica de la fábrica

(***) El intervalo dado es compatible con los contenidos de $\mathrm{CO}_{2}$. 
longitud, se concibe llegar a producir 3.000 t de cal por día, a partir de un sistema de horno rotatorio único. Los hornos rotatorios son versátiles; pueden manejar un tamaño de alimentación hasta de $5 \mathrm{~cm}$ y relaciones hasta de 1:3. Son capaces de calcinar un producto alimentado en pasta o tortas procedentes de filtros en húmedo, y pueden quemar todos los combustibles disponibles, solos o en combinación entre ellos: gas natural, gases de procesos, fuel-oil y carbón pulverizado. Puede producirse una amplia gama de calidades de cal, desde cales calcinadas a muerte a cales de gran actividad, con contenidos de $\mathrm{CO}_{2}$ comprendidos entre 0,2 y $0,8 \%$. Es posible la eliminación de azufre, sin afectar materialmente otras características, manteniendo adecuada temperatura, adecuada atmósfera del horno y controlando la cantidad de polvo recuperado y reintroducido al sistema.

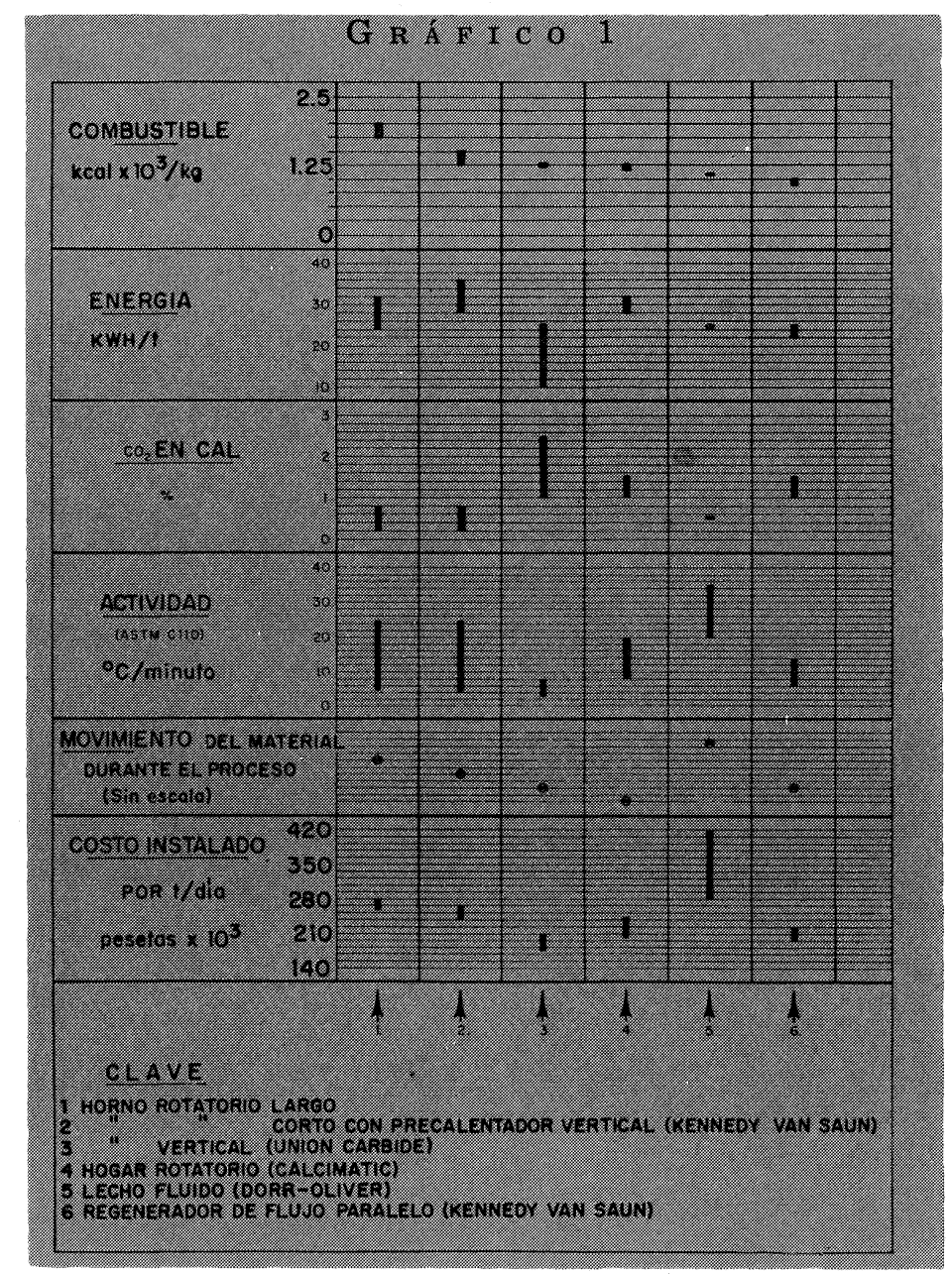

A medida que se estrecha el margen en el tamaño de la alimentación, y el tamaño mínimo está por encima de $25 \mathrm{~mm}$, un elevado grado de mezcla en el lecho durante la calcinación produce una cal muy uniforme. El elevado movimiento del lecho de material contribuye a la abrasión y formación de polvo y, por tanto, las exigencias de depuración son grandes.

El consumo de combustible de los hornos rotatorios es, generalmente, elevado; por encima de 1,75 a $2,00 \times 10^{3} \mathrm{kcal} / \mathrm{kg}$; el consumo de energía oscila de 24 a $32 \mathrm{kWh} / \mathrm{t}$, dependiendo principalmente del tipo de sistema de combustión y de las exigencias de recupe- 
ración de polvo. Las necesidades de espacio son muy grandes; los hornos de cal existentes hoy día tienen hasta $120 \mathrm{~m}$ de longitud. Los hornos rotatorios son caros; los costos de equipo para los sistemas de calcinación oscilan de 105.000 a 125.000 ptas./toneladas diarias de producción. Los costos del equipo instalado oscilan de 260.000 a 280.000 ptas./toneladas diarias de capacidad.
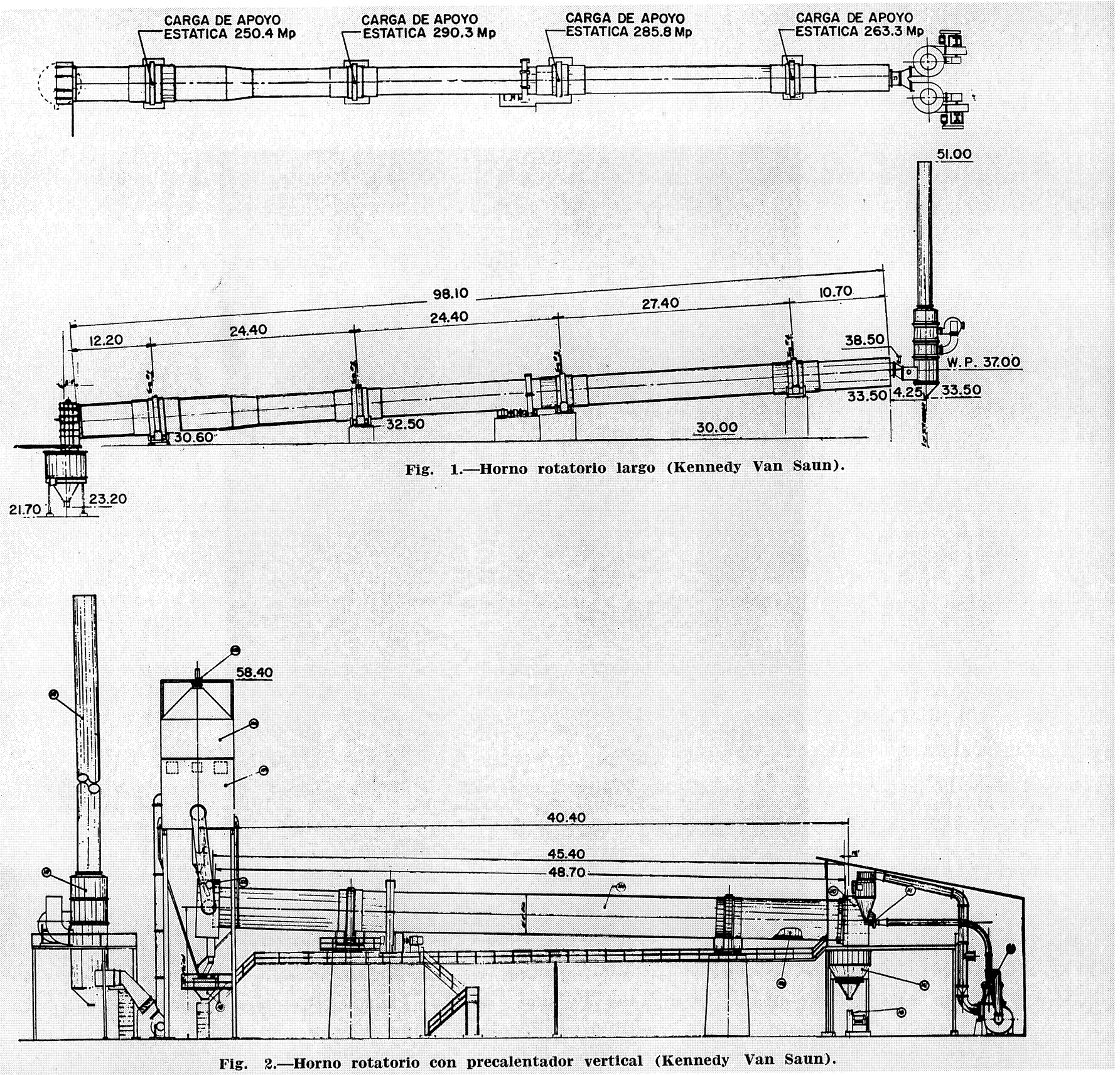

Las exigencias para el equipo de recuperación de polvo varían con el tamaño de alimentación y la calidad de la materia prima. Generalmente son grandes porque el volumen de gases es elevado; puede ser preciso el templado con aire o agua; son normales dos 
pasadas y los hornos de pasta con tamaño de alimentación fino presentan cargas de polvo en los gases de escape más elevadas.

El equipo de enfriamiento empleado en los hornos rotatorios es generalmente de uno de los dos tipos siguientes: enfriadores satélites para materiales finos o enfriadores del tipo de contacto para cal gruesa. Los enfriadores satélites tienen menos recuperación de calor, pero también menos costos de marcha y conservación; en tanto que los enfriadores de contacto tienen un mejor comportamiento calorífico, pero los costos de operación son más elevados y las necesidades de espacio apreciables. Los enfriadores rotatorios y los de parrilla son secundarios, a la hora de la elección, en la industria de la cal.

\section{HORNOS ROTATORIOS CORTOS CON PRECALENTADORES EXTERNOS}

Se emplean dos sistemas básicos: los precalentadores verticales (de Kennedy Van Saun Corp.) y los precalentadores de parrilla (de Allis-Chalmers). En ambos sistemas, los gases de escape del horno rotatorio corto entran a los precalentadores a temperaturas que oscilan de $925^{\circ}$ a $1.150^{\circ} \mathrm{C}$, y parte de la calcinación ocurre en el precalentador. Los precalentadores verticales pueden, por su diseño refractario, recibir gases a mayores temperaturas y, por tanto, tener mayor economía de combustible y costos de conservación más bajos. Los precalentadores de parrilla pueden tener quizás un margen más amplio de tamaños de alimentación. Presentan una caída de presión menor, pero requieren aleaciones moldeadas y, por consiguiente, están limitados en las temperaturas permisibles a los gases de escape y son más vulnerables a oleadas de temperatura imprevistas.

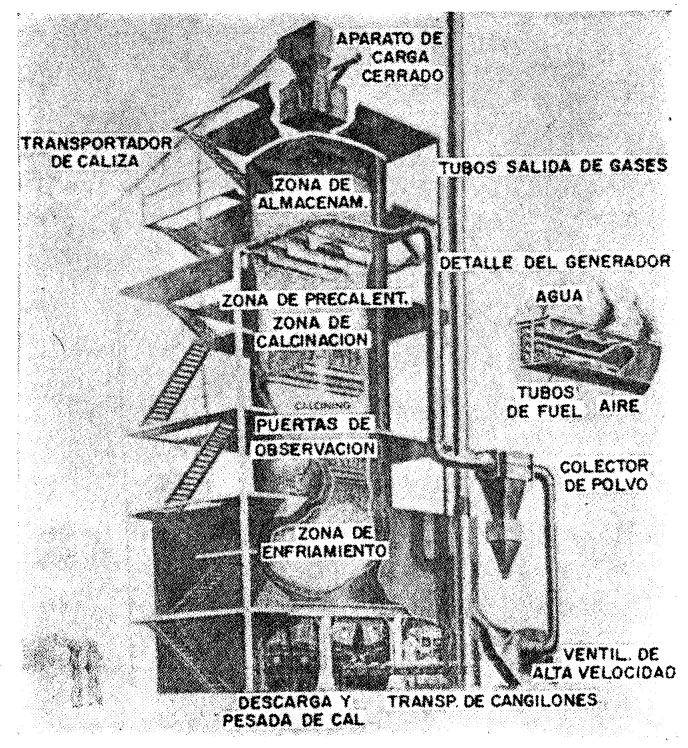

Fig. 3.-Horno vertical (Unión carbide).

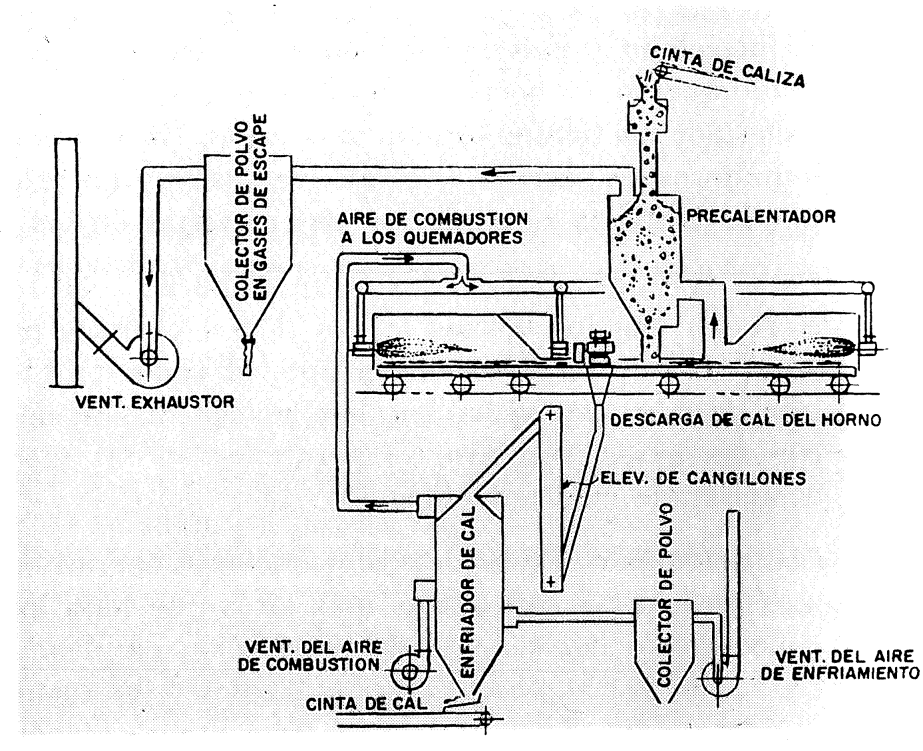

Fig. 4.-Hogar rotatorio (Calcimatic).

La capacidad de estos sistemas es elevada, similar a la de los hornos rotatorios largos, pero el tamaño máximo de las unidades presumiblemente se mantendrá por debajo a causa del diseño del precalentador.

Los tamaños de alimentación aceptables son más limitados que para los hornos rotatorios largos; usualmente el tamaño mínimo es $10 \mathrm{~mm}$ y el máximo $45 \mathrm{~mm}$, siendo la re- 
lación permisible de 1:4. Pueden emplearse todos los combustibles disponibles y sus combinaciones, y las calidades del producto son idénticas a las de las cales obtenidas en horno rotatorio largo.

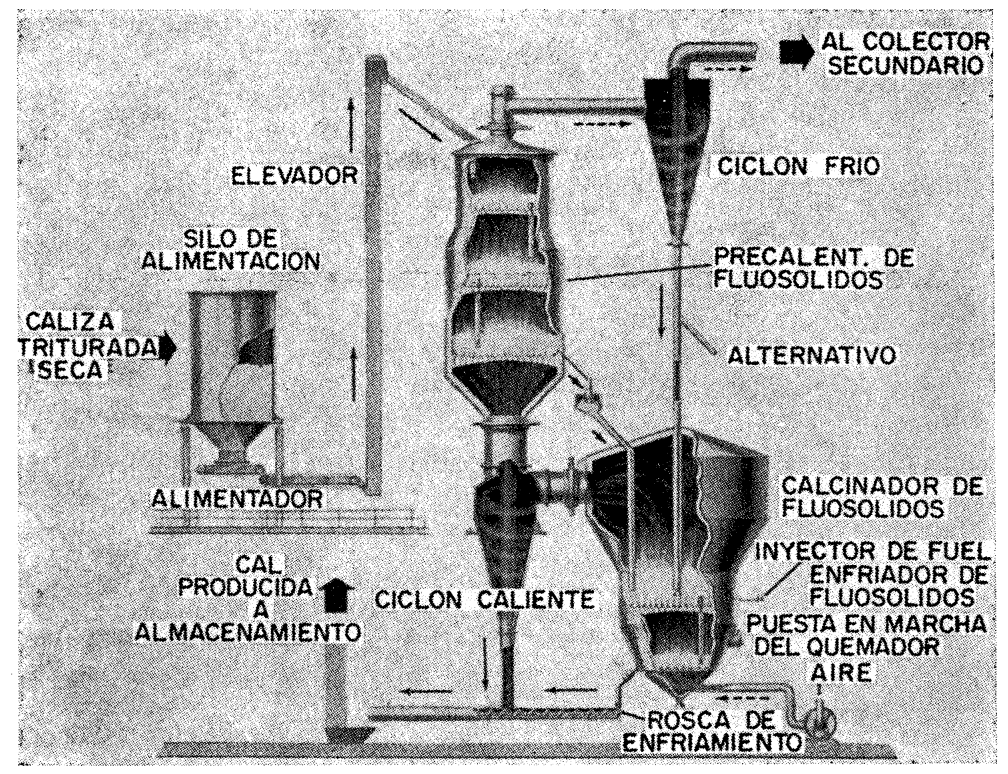

Fig. 5.-Lecho fluido (Dorr-oliver).

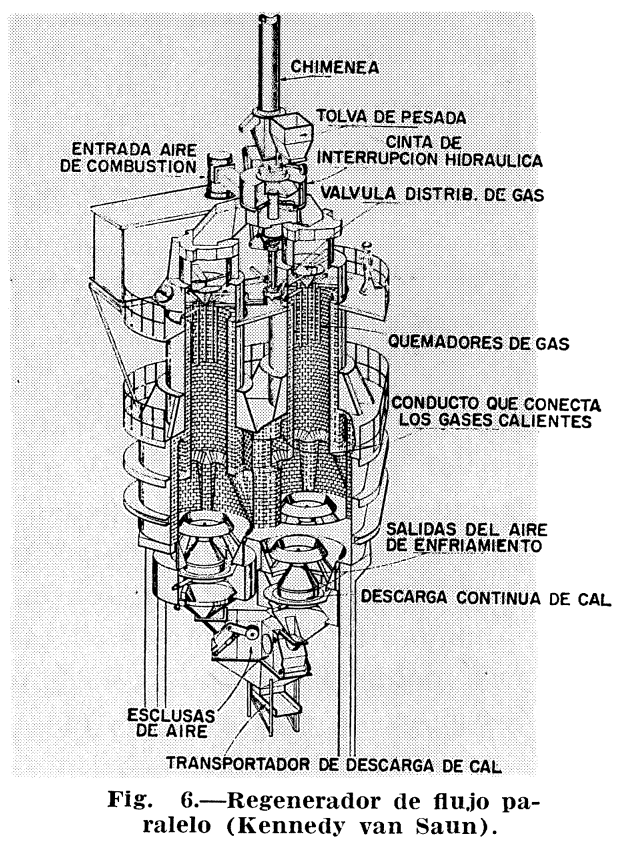

ralelo (Kennedy van Saun).

El mezclado y movimiento del lecho en la zona de calcinación tienen lugar en el mismo grado que en los hornos rotatorios largos, aunque el material se expone al movimiento del lecho por un tiempo mucho más corto. La alimentación permanece inmóvil en los precalentadores de parrilla, y se mueve suave y muy lentamente en el precalentador vertical; por lo tanto, la degradación del material en los sistemas con precalentador es reducida.

Como la transmisión de calor en el horno rotatorio se produce en gran medida por radiación, y como los hornos largos tienen grandes pérdidas por radiación en la superficie y pérdidas por los gases de escape, al reducir la longitud del horno y sustituir la transmisión de calor por radiación, por la transmisión de calor por convección y contacto directo con los gases, resultan temperaturas en los gases de escape más bajas y consumos de combustible más reducidos.

Los sistemas con precalentador eficiente operan entre 1,25 y $1,5 \times 10^{3} \mathrm{kcal} / \mathrm{kg}$; los consumos de energía son quizás más altos que para los hornos rotatorios largos, 32 a $36 \mathrm{kWh} / \mathrm{t}$, dependiendo del sistema de calefacción empleado. Los costos de las inversiones son de 100.000 a 120.000 ptas./toneladas diarias y los costos del equipo instalado oscilan de 240.000 a 270.000 ptas. Estos costos son para precalentadores de tipo vertical; para los precalentadores de parrilla resultan quizás más elevados.

Las necesidades de espacio para los sistemas con precalentador son pequeños, del orden del 60 al $70 \%$ de las necesidades para un horno rotatorio largo. Los sistemas de horno con precalentador son particularmente afortunados cuando se equipan con enfriadores de tipo de contacto y fosos de precalentamiento. La aplicación de los sistemas 'con precalentador se limita a materiales de alimentación que no decrepitan o degradan durante la calcinación. 
Las necesidades de colección de polvo para los sistemas con precalentador son reducidas a causa de las menores cantidades de gas que se manejan, resultado de un menor consumo de combustible y de una temperatura más baja de los gases de escape; las cargas de polvo son generalmente inferiores porque los precalentadores actúan como filtros de polvo. Esto es particularmente cierto en los precalentadores de tipo vertical por su mayor altura de lecho.

\section{HORNOS VERTICALES}

Se han proyectado y están en marcha muchas variaciones del horno de tipo vertical. La mayor parte de las versiones más sofisticadas en Europa se han desarrollado en hornos de gran capacidad por los costos de combustible apreciablemente más elevados; sin embargo, la mayor parte de la cal europea procede de hornos de alimentación mixta y, en consecuencia, es de inferior calidad. Es fuertemente calcinada, alta en núcleo e impurezas procedentes del coke del combustible. Los hornos verticales calentados con aceite o gas con muy bajos consumos de combustible $-0,9$ a $1,1 \times 10^{3} \mathrm{kcal} / \mathrm{kg}$ - se han desarrollado en Europa. Entre ellos se encuentran: el horno Beckenbach, el horno de flujo transversal Heiligenstaedt, los hornos con secciones verticales inclinadas, las secciones transversales anulares y el empleo de circulación de los gases de escape. En la industria de la cal americana sólo han encontrado aplicaciones los proyectos de Azbe; tienen un intervalo de capacidad limitado y no se considerarán en este trabajo. De considerable interés comercial en los Estados Unidos es el horno vertical de gran capacidad de la Unión Carbide Corp., del cual referiremos los siguientes datos:

Es disponible en capacidad desde 100 hasta 680 t/día, emplea combustible gaseoso, y el tamaño de la alimentación oscila de 2,5 a $17,5 \mathrm{~cm}$, con una relación permisible de $1: 2$. El consumo de combustible es de $1,25 \times 10^{3} \mathrm{kcal} / \mathrm{kg}$ y el consumo de energía oscila de 10 a $25 \mathrm{kWh} / \mathrm{t}$, dependiendo del tamaño del horno. La calidad de la cal para una operación normal oscila de 1,0 a $2,5 \%$ de $\mathrm{CO}_{2}$ y la actividad de la cal se encuentra en la zona media.

El material en la vertical se mueve lentamente y prácticamente no ocurre ninguna mezcla durante la calcinación. A causa de su gran producción específica, se emplean temperaturas de calcinación elevadas y es posible alguna canalización de los gases. Por el lento movimiento del lecho es de esperar una uniformidad más baja en la cal viva, frente a los sistemas de calcinación con un movimiento elevado del lecho. A causa del mayor tamaño de alimentación, las necesidades de colección de polvo se realizan en dos fases. La carga de polvo en los gases de escape oscila de 5,5 a $10 \mathrm{~g} / \mathrm{m}^{3}$ normal. Las exigencias de espacio son pequeñas.

\section{CALCINADOR DE HOGAR ROTATORIO}

Este sistema es fabricado por Calcimatic Ltd. Se caracteriza por la total ausencia de movimiento de la piedra (o cal) durante la calcinación y por mínimos movimientos durante el precalentamiento y el enfriamiento. Pueden manejarse piedras muy blandas sin producir apreciable cantidad de finos. Por la ausencia de movimientos, el lecho de piedra sobre el hogar no puede exceder ciertos límites en la altura y distribución de tamaños y son posibles variaciones en la calidad de cal desde el fondo a lo alto del lecho. Existen unidades en tamaños que oscilan de 100 a 600 t/día, el consumo de combustible es de 1,18 a $1,25 \times 10^{3} \mathrm{kcal} / \mathrm{kg}$ y son posibles de emplear combustibles líquidos y gaseosos. El consumo de energía es de 28 a $32 \mathrm{kWh} / \mathrm{t}$. El producto es una cal altamente reactiva con 1,0 a $1,5 \%$ de $\mathrm{CO}_{2}$ y un tiempo de reacción total de 3 minutos, de acuerdo con ASTM C 110. La carga de polvo en los gases de escape es baja. Las necesidades de espacio osci- 
lan desde 5,5 a $12,0 \mathrm{~m}^{2}$ y los costos del equipo instalado son de 200.000 a 250.000 ptas./toneladas diarias.

\section{LECHO FLUIDIZADO}

Estos sistemas los suministran Dorr-Oliver y Fuller Co. Los calcinadores de lecho fluidizado han encontrado una aplicación limitada en la industria de la cal y en las plantas de recalcinación de lodos de cal por sus estrechas exigencias en el tamaño de la alimentación. Son particularmente adecuados cuando se dispone de alimentación fina (procedente del proceso de trituración de piedra) o cuando la piedra rompe preferencialmente a un cierto tamaño de grano por su estructura cristalina o hendidura.

Se dispone de calcinadores de lecho fluidizado en tamaños que oscilan de 50 a $300 \mathrm{t} /$ día (de Dorr-Oliver), es posible la calefacción con gas o aceite y el consumo de combustible es de $1,05 \times 10^{3} \mathrm{kcal} / \mathrm{kg}$; el consumo de energía es de $25 \mathrm{kWh} / \mathrm{t}$.

Por el violento movimiento del lecho, se obtiene un producto altamente reactivo. Es posible que las cales más reactivas procedan de los sistemas de lecho fluidizado. Los contenidos de $\mathrm{CO}_{2}$ llegan a bajar hasta $0,5 \%$. Por el movimiento tan violento del lecho, la degradación del material de alimentación puede ser un problema y las cargas de polvo en los gases de escape resultan muy altas. El tamaño de alimentación preferido se encuentra entre 0,25 y $6,00 \mathrm{~mm}$, aunque puede manejarse material entre 0 y $6,00 \mathrm{~mm}$. Las unidades de lecho fluidizado requieren poco espacio en superficie, pero son altas y similares a los hornos verticales. El costo del equipo instalado es elevado -el más alto de todas las unidades de calcinación de cal-y oscila entre 280.000 y 420.000 ptas./toneladas diarias de producción.

\section{HORNO RECUPERADOR DE FLUJO PARALELO}

Este sistema se desarrolló en Austria y existen actualmente unos 60 hornos en marcha o en construcción. En Norteamerica lo construye Kennedy Van Saun Corp. (horno vertical multicolumnar). El principio de la calcinación en flujo paralelo para obtener una cocción blanda se combina con el precalentamiento del aire de combustión, lográndose así un consumo de combustible más bajo a la vez que se mantiene una gran calidad y actividad en la cal. Por su diseño de horno vertical, solamente ocurre un pequeño movimiento de la piedra o de la cal en el lecho. El sobrecalentamiento local y la sobrecocción se evita porque las partes más calientes y los gases de combustión se hallan solo en contacto con la piedra que rápidamente absorbe el calor a alta temperatura, por emplearse un flujo paralelo.

El sistema existe en capacidades que oscilan entre 200 y 400 t/día y el tamaño de alimentación aceptable fluctúa entre 20 a $150 \mathrm{~mm}$, con una relación no superior a 1:3. El consumo de combustible es de 0,9 a $1,0 \times 10^{3} \mathrm{kcal} / \mathrm{kg}$ y el consumo de energía oscila entre 22 y $25 \mathrm{kWh} / \mathrm{t}$. La calidad de la cal es de 1,0 a $1,5 \%$ de $\mathrm{CO}_{2}$ y la actividad es alta, oscilando entre 3 y 5 minutos el tiempo total (ASTM C 110). Las exigencias de espacio son pequeñas, $18 \times 18 \mathrm{~m}$ como máximo, pero los hornos son altos. Normalmente estos hornos se calientan con gas, aunque existen en funcionamiento un pequeño número de hornos calentados con fuel-oil. El costo del equipo es de 125.000 a 140.000 ptas. y el costo instalado oscila entre 200.000 a 225.000 ptas./toneladas diarias.

Las características más importantes y los datos de operación de estos seis sistemas de preparación de cal se muestran en la tabla I y en el gráfico I.

Los datos y costos de marcha fueron suministrados por Calcimatic Ltd., Dorr-Oliver Inc. y Unión Carbide Corp. 